\title{
Helping older people to manage their social activities at the retirement home
}

\author{
Benoît Otjacques, Marc Krier, Fernand \\ Feltz \\ Public Research Centre - Gabriel Lippmann \\ "Informatics, Systems and Collaboration" \\ Department \\ 41, rue du Brill \\ L-4422 Belvaux (Luxembourg) \\ \{otjacque, krier, feltz\}@lippmann.lu
}

\author{
Dieter Ferring, Martine Hoffmann \\ University of Luxembourg \\ INSIDE Research Unit \\ Route de Diekirch \\ L-7220 Walferdange (Luxembourg) \\ \{dieter.ferring, martine.hoffmann\}@uni.lu
}

\begin{abstract}
This paper discusses the early results of the multidisciplinary project TIVIPOL aiming to propose digital technologies to enhance the social life of older people in a retirement home. A prototype combining a tactile color screen, a RFID reader and a ticket printer is described. It allows the older people to manage their participation to the social activities organized by the home staff. A first evaluation has shown the usability as well as the good acceptance of this system.
\end{abstract}

\section{Categories and Subject Descriptors}

K.4.2. [Computer and Society]: Social Issues - Assistive technologies for persons with disabilities

\section{General Terms}

Human Factors

\section{Keywords}

Older people, Tactile, RFID, Field Study

\section{INTRODUCTION}

Numerous researchers have investigated how Information and communication Technologies (ICT) can be used by older people. Several major trends can be identified in this context. Some initiatives try to maintain older people at home with a good quality of life and an appropriate safety level (e.g. [1], [2]). Another trend aims to limit the consequences of the sensory or cognitive impairments coming with ageing (e.g. [3] for people with dementia). Other researchers focus on the medical support (e.g. [4], [5], [6]). Those applications differ from several aspects: the type of support provided to the elderly (e.g. social, psychological, physical level); the intended place of use (e.g. at home, in a care institution, at the hospital) or the technology used (e.g. sensors, tactile screens, mobile phones).

Our research focuses on supporting the social life of the residents of a retirement home with ICT devices. We try to design objects that are seen as innovative ICT-related objects

(C) The Author 2009.

Published by the British Computer Society but not as computers and we study the design factors that favor or hinder the acceptation of these technologies by the older population.

It is often claimed that the elderly people feel uneasy with computers and ICT technologies (cf. e.g. [4]). In many cases, this is due to the fact that those devices do not take into account the age-specific changes in sensory, motor and cognitive abilities. From another perspective, the concepts, habits and experience of the ICT professionals significantly differ from those of the elderly people. This dichotomy may result in negative experiences and (justified or unjustified) mistrust in ICT technologies by the latter. Our purpose is to try to reduce the gap between those two worlds. We want to keep visible the ICT nature of the devices that we design for older people but to adapt these objects to their needs and skills. Previous research (e.g. [7]) has shown that under these conditions older people are not adverse to new technologies.

\section{TECHNOLOGY AND AGEING}

Gerontechnology can be defined as the scientific discipline studying the factors ensuring an optimal technological environment of all aging and old people up to a high age. Bouma et al. [8] point out that gerontechnology has to intervene in five domains of daily life: health and self-esteem (D1), housing and daily living (D2), mobility and transport (D3), communication and information (D4), and work and leisure (D5) with four goals for development: enhancement and satisfaction (G1), prevention and engagement (G2), compensation and assistance (G3), and care support and organization (G4).

The research described in this paper mainly aims to support daily living and self-esteem for older people in good physical and mental conditions. Nevertheless even people getting older without severe impairments or dementia can face complicating factor when they have to use innovative technologies.

The individual characteristics of the user, the technological features of the device (see [7] for a comparative evaluation) and the interaction process all play a role in this context (see [9] for an overview). In addition to the physical or cognitive ability to use a new device, the individual motivation is also a critical factor to take into account. Therefore the adoption process must be carefully understood at the individual but also at the larger social level. The degree of similarity and compatibility with existing knowledge and skills must also be mentioned here. Reducing the learning period is acknowledged as a positive element to increase the potential adoption of any new ICT technology. 
An additional challenge in gerontechnology consists in making the computer scientists aware of the specific skills of the older people. From this perspective, the dimension of control, i.e. the feeling of being able to steer the technology under question and to avoid negative effects is often underestimated. Finally emotion also seems to influence the adoption of a new system. However it has been observed that some interfaces essentially relying on emotional interaction can be rejected by older people (e.g. [10]).

\section{TIVIPOL PROJECT}

The TIVIPOL research project was launched in fall 2007 with the purpose to investigate how older people accept new ICT technologies in retirement homes. It regroups computer scientists and psychologists specialized in gerontology. As the project evolved the mixed nature of the project team clearly appears as an advantage. The computer scientists' temptation to design and develop some systems based on cutting edge technologies is tempered by the psychologists who insist on the need to take into account the specific cognitive and social features of the older people. Symmetrically the psychologists are made aware of some technological opportunities but also of the limitations of the current state of computer science.

A retirement home in Luxembourg (called " $\mathrm{K}$ " in this paper) was selected for our pilot study. This institution was chosen because its clientele mainly comprises seniors of comparable good health who live autonomously in apartments located within the same building. There are 118 residents in this home. They have no severe health related problems (i.e. health-care requirements of the residents must not exceed 12 hours per day).

\section{DESIGN RATIONALE}

Our design process is built upon former research on the adoption of ICT systems. This topic has been largely studied in the management of information system (MIS) literature. While the TIVIPOL project focuses on a completely different target group (older people of a retirement home instead of managers or office workers), we took into account the results of MIS researchers. The technology acceptance model (TAM) [11] explains that the perceived usefulness and the perceived easeof-use are major factors influencing the adoption of new ICT systems. The Unified Theory of Acceptance and Use of Technology [12] claims that performance expectancy, effort expectancy, social influence, and facilitating conditions determine the usage intention and the user behavior.

Combined with findings from the gerontology research (e.g. [9]) these elements allowed us to identify ten key design rules (KDR) for the new system: to fulfill existing real needs of the users (KDR1); to offer some advantages for the retirement home staff (KDR2); to require a very short learning phase (KDR3); to require no previous skill in computer usage (KDR4); to have a personalized user interface (KDR5); to be enjoyable to use (KDR6); to support social life (KDR7); to rely on a pleasant, reliable and robust terminal (KDR8); to be able to evolve progressively according to individual pace (KDR9); to support functional developments without extensive resources (KDR10).

These rules are intended to facilitate the final adoption of the system and make it become a success story. In fact, these key factors are well known in the literature and should always be taken into account. However, they are critical in our case due to the specific nature of the target population. The main goal is to persuade the older people that they are able to use efficiently an ICT-based system, providing that it has been carefully designed to take care of their own characteristics. In this context we were especially careful about the first impression given by the new system. From this perspective the lack of maturity that many research prototypes must admit (often due to a lack of resources that are available for the development phase) could potentially play a negative influence in our case. Therefore we passed several months to design and build an initial version of good quality before its deployment in the "K" home. Nevertheless, some formative evaluations were carried out during the design and development process. They involved a small subset of the real users in dedicated evaluation sessions. We adopted this approach to avoid as much as possible the elements that could potentially generate fear or distrust in the initial version of the new system (e.g. software bugs, fragile hardware or unexpected behavior). We put the priority on simplicity and stability instead of complexity. This approach is also critical in our case for another reason. As the system is intended to be used in a longitudinal field study in the " $\mathrm{K}$ " home, it is important that it does not require a permanent presence of computer scientists to be used in practice.

\section{PRELIMINARY FIELD STUDY}

\subsection{Discussions with the " $K$ " staff}

In order to identify the features that the new system should integrate, the TIVIPOL project team discussed with the manager of the " $\mathrm{K}$ " retirement home. He explained that the home residents can participate to several activities organized by the home staff. For instance, they can choose the lunch menu for the next days, visit some exhibitions in the city, go shopping by coach, or participate to arts and crafts activities. Generally the residents must register at the home entry desk or via the home staff to attend these activities. To the limit of our knowledge, little research explored how older people can personally manage their social activities in a retirement home. The organization of time-related tasks often targets other issues (e.g. self-management of health [7], [13]). This aspect of the residents' life was then selected to be handled via innovative ICT technologies.

The reservation of the menus at the cafeteria was chosen as the first use case of our system. Several arguments were underpinning this decision. Lunch time is a very important moment of the day for the residents from a social point of view. It is also a frequent subject of discussion among the residents. Moreover, most of the residents are involved in the selection of the menus, which is not the case of the other activities. For instance, they are not equally interested in art exhibitions. We did not want to exclude some residents on the basis of their individual preferences (e.g. visits of exhibitions, shopping, gardening...). The selection of the menus is also a well known process for every resident. It does not require a learning phase that would overlap with the discovery of the new system. Generally speaking it appears as a promising starting point. Of course, it must be considered as a first step. The system is intended to support a much larger range of social activities but at this stage we don't want to worry the residents with a complex set of new proposals regarding many of their daily activities.

Before the TIVIPOL project, the residents were receiving a paper form to select their menu for the coming days. They could also directly order at the cafeteria when they come for 
lunch. In this case, however, they are not certain that the dish that they prefer is still available.

The system purpose is to allow the residents of the " $\mathrm{K}$ " home to manage their registrations to the activities (reservation of the menus in a first step, others activities in subsequent stages) organized in the " $\mathrm{K}$ " home. This objective is consistent with two of our key design rules. First, the system simply offers a new optional way to carry out some existing activities of the older people (cf. KDR 1). Second, a better management of the registrations can help the home staff to plan and organize the activities (cf. KDR2). Indeed, before the TIVIPOL project, the home staff did not have a complete overview of the registrations because they were manually processed and the relationships among the participations to each activity were not easy to identify. The new system will also offer additional convenience to the older people (e.g. they can handle their registrations whenever they want, the opening hours of the entry desk are not important any more). From a psychological viewpoint, it might also make the older people more aware of their ability to master an IT-based system, which could increase their self confidence. If this hypothesis was confirmed, the system would probably provide both satisfaction and pride to the concerned residents.

\subsection{Study of the target population}

In order to get a first description of the target population, semistructured interviews have been conducted at the initial phase of the project. In detail, social networks and social support resources have been explored. Furthermore, attitudes towards and experiences with modern technologies have been studied. A random sample of $n=40$ elderly people has been interviewed by trained student interviewers in two different institutions (including "K"). In both cases, home managers facilitated the recruitment of the sample and further supported the project and its implementation in their institutions. Participants were on average 78.6 years old with a slight overrepresentation of women $(57 \%)$ in the sample. None of the participants had a severe psycho-physical or functional condition. Results of this preliminary study showed that the elderly used in first place telephone calls for social contact and they reported in general to have little or no experiences with modern technologies such as the internet and mobile phones. On the other hand, attitude towards modern technologies in general were quite positive and accepting.

These findings seems to confirm the argument claiming that the difficulties encountered by older people to use modern technologies do not result from an a priori opinion but rather from inappropriate design.

\section{SYSTEM DESCRIPTION}

\subsection{Hardware}

From a conceptual viewpoint, the hardware used in our system is composed of three elements: an 8.4' tactile color screen, a RFID reader and a ticket printer (cf. Figure 1). They are described in this section.

In order to satisfy (KDR4) we have decided to use a terminal physically different from a classic computer. This approach is justified by the observation that most of the " $\mathrm{K}$ " home residents do not use a computer at all. The (KDR8) constraint led us to contact a professional OEM manufacturer to configure a device that does not look like a fragile research prototype. In cooperation with this company we configured a terminal including a tactile color screen, a RFID reader, a WIFI connection, and which is able to run Java applications and to connect to a database via Internet.

The RFID technology is used to support the identification of the users (i.e. how to $\log$ into the software application). An individual RFID tag is distributed to each user who can choose its physical aspect (key ring, card similar to a credit card or tag attached to a personal object). Two elements underpin our decision to adopt this technology.First, the RFID technology is nowadays mature and reliable (cf. KDR8). Second the learning time required to use it is very short (cf. KDR3).

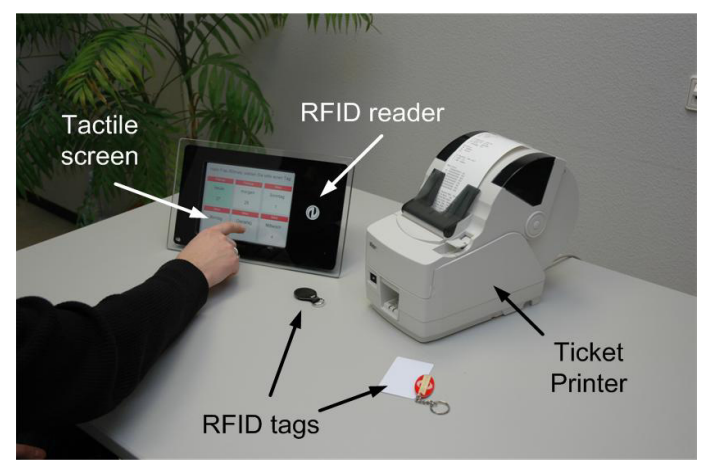

Figure 1. Complete system

The interaction with the application relies on a tactile color screen. This technology is also popular for several years and is well known even by older people (cf. KDR4). Indeed, it is frequently used in many circumstances of the common life (e.g ATM, public transportation terminals). In this context, it must be reminded that the greatest challenge concerns the graphical design of the user interface and the navigation into the application.

For providing to the home residents a tangible feedback of their registrations, a specific ticket printer will be placed near the terminals in the entry hall. The older people will receive a ticket for each registration. The name of the resident and the registration details are printed on the ticket (e.g. day, chosen dishes). This feature plays several roles: memory aid; reassuring function to confirm that everything was correctly processed; tangible proof of registration for the activity organizer.

\subsection{Software}

The first step to use the application is to log in with one's personal RFID tag. In order to keep this operation as simple as possible an animation showing how to use the RFID tag is continuously displayed on the terminal screen when it is in rest mode. This feature reminds to the users how to use the system (cf. KDR3, KDR4). Moreover the dynamic nature of the animation acts as an attractor for the people passing near the terminal (cf. KFR7).

The design of the software user interface was quite challenging. Indeed, many daily users of computers take it for granted to have a "close window" or a "cancel" button in every window. However, previous research [14] has pointed out that when designing interfaces for specific categories of users (e.g. older people) it can be advantageous not to follow some legacy paradigms like WIMP (Window, Icon, Menu, Pointing device). On this basis and considering the (KDR4) factor, the user interface was completely designed to take into account the specific knowledge of the older people and the context of the retirement home. 


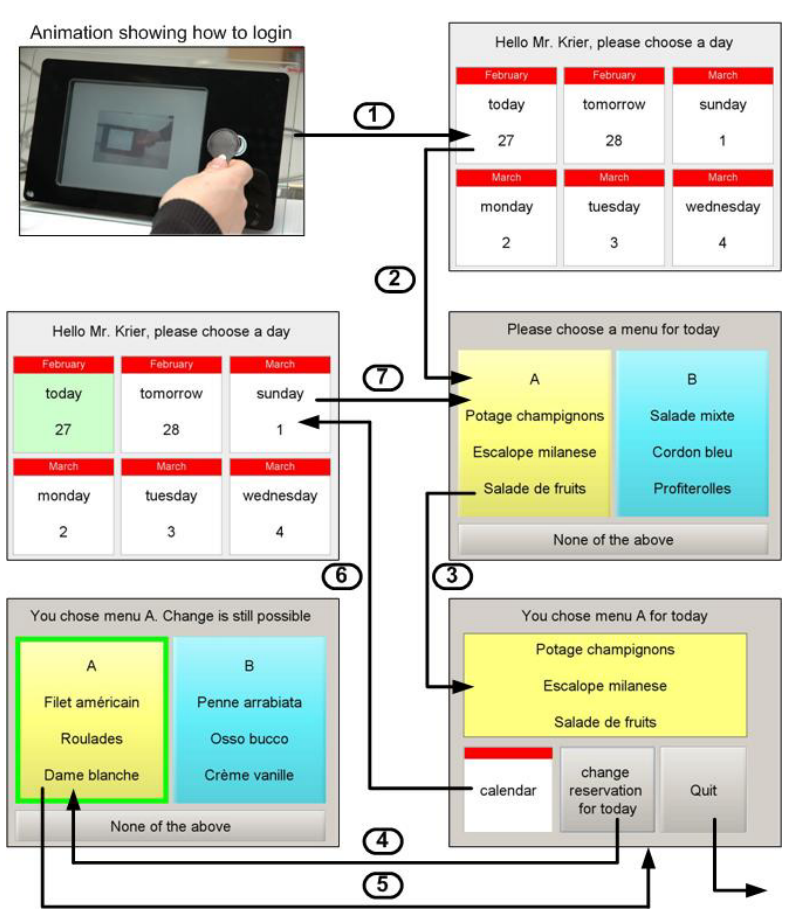

Figure 2. Navigation into the application

Once the user is connected very simple screens are displayed. The navigation has been designed to be very intuitive. (cf. Figure 2). Choosing the day of the reservation is the (1) step after login. The overall look of this screen mimics a classic calendar. This metaphor is well known by older people (cf. KDR3). Note that the days are all identified in an absolute manner (e.g. $27^{\text {th }}$ of February). However, the current day and the day after are also named in a relative manner ("today", "tomorrow"). This feature has been adopted to lower the cognitive load for the older people. It is much easier to remember that current day is "today" than it is "Friday".

Pushing on the button of the selected day (2) opens a new screen where the two menus available at the cafeteria are displayed. The user select his/her preferred one by pushing on the appropriate button. Then a new screen reminds the selected choice (3). The user can then change its reservation (4), reserve for another day (6) or quit the application.

The user interface was specifically designed to remain as simple and sober as possible. Neutral colors were chosen. Simple and explicit labels are used. This approach was motivated by the fact that most of the older people prefer a calm and pleasing environment.

The labels on the buttons and the informative text have been chosen with the user viewpoint in mind. They do not use the standard names of classic applications (e.g. "close", "next", "exit"). We rather use some sentences (e.g. "Please choose a menu for today") that are easy to understand for an older person without any previous skill in using a computer (cf. KDR4).

In every screen the current state of the application variables is reminded. For instance, the day concerned by the reservation or the selected lunch menu is displayed on top of the screen (e.g. "Please choose a menu for today", "You chose menu A"...). This feature is usually recommended in every user interface. However, it is of outmost importance for older people to avoid the feeling of being lost in the application (cf. dimension of control). From this view point it was also critical to explicitly mention in the screen the possible behaviors that the system accepts (e.g. "Change is still possible" after the selection of a lunch menu).

We also took care of keeping an overall coherence of the colors used in the user interface. Each lunch menu is always colored with the same color (e.g. yellow or blue). The green color is always associated with a selection already made in the system (e.g. green border around the selected lunch menu, green background in the calendar for the days with an active reservation).

In addition, according to (KDR5), the application is fully multilingual because the home residents potentially use three languages (French, German, and Luxembourgish). Just after the login the user is personally welcomed in the application in his preferred language. In the future it is also planned to communicate some personal information via the terminal.

At this stage of the project the reservation is available for one specific activity (lunch menus). However, this module is generic and can be easily completed to handle others types of registrations according to the feedback of the evaluation process (cf. KDR10).

\section{FORMATIVE EVALUATION}

\subsection{Methodology}

The acceptance by the end-users of the new application described in this paper (tactile terminals + RFID; ticket printer not included in this evaluation) was evaluated with a combined methodology: an observational study and an evaluative posttest interview.

A total of $n=19$ participants were pre-selected and recruited by the " $\mathrm{K}$ " home manager who also arranged appointments for individual test sessions with the elderly. In this sample four persons also participated in the first interview-study. The participants were on average 84 years old, mainly female $(68 \%)$, mostly widowed $(84 \%)$, with low or no experience or interest in modern technologies. Participants were living in the retirement home for six years on average. None of them suffered from severe psycho-physical deficits or functional impairments.

The evaluation sessions were carried out in the " $\mathrm{K}$ " home in order to keep the subjects in their usual environment (i.e. avoid stress and troubles due to the laboratory effect). In addition, the system is evaluated in the context of its future use, which should strengthen the validity of the results (cf. ecological evaluation).

The one-by-one testing methodology was chosen in order to preserve or maximize the internal validity of the study. The participants received standardized information on the purpose and functioning of the prototype, as well as standardized operating instructions (e.g. to make reservation for two consecutive days, to cancel a reservation and to change the menu). Residents tested the new application during a 20minutes testing phase and were subsequently interviewed about their experience by two trained interviewers.

Note that the system was loaded for each subject with his/her personal data (e.g. real name, preferred language) and realistic lunch menus. This option was chosen to avoid biases in the usability study. Indeed, Genov et al. [15] have pointed out the potential biases due to unrealistic data loaded in the system to be evaluated. 
The same two interviewers accompanied and monitored the older adults during their first contact and use of the application. Thereby, they assessed cognitive, behavioural and emotional reactions of the participants while handling the new device. Subsequent to the hands-on testing phase, the participants were queried with respect to the following aspects:

- Appraisal of the manageability, usability and the overall acceptance of the new technological device in the setting of a retirement home;

- Evaluation of the design-related and functional characteristics of the prototype by the seniors;

- Suggestions for improvement from the perspective of the older adults.

\subsection{Results}

According to the observations of the two interviewers, most participants understood the purpose of the study from a cognitive point of view and had no difficulties in understanding the application of the new technical device. Three persons, however, had problems to concentrate on the task and carry out the instructions. All in all, interviewers' observations indicated that participants dealt in a very confident way with the machine; few persons exhibited nervousness, concentration difficulties or disinterest. Furthermore some participants made fruitful suggestions for the further improvement of the device.

Results of the subsequent interviews showed in general a good acceptance, practicability and applicability of the new device. On a 4-point Likert-scale, participants evaluated the system on average as "quite" user-friendly, manageable, useful and potentially time-saving. The range of the response profiles indicate however that opinions do differ between the participants and that not everyone shares the overall positive attitude (e.g. "I don't want this machine, I prefer to order my meal the usual way"). Referring to the question whether they would like to use the machine in the future to reserve their weekly menus half of the residents agreed, whereas five were undetermined and four rather disagreed.

With regard to design-related and functional characteristics the residents evaluated all the cited aspects as "good" or "very good" (in detail: font size, font color, background color, display size, illustration of the lunch menu, illustration of the weekly calendar, visibility of the reservations). However, some of them mentioned that the font size should be modifiable for persons suffering from amblyopia. We also observed that a small number of subjects had some difficulties to correctly operate the tactile screen. They touch it several times at high frequency, which do not give them the opportunity to really see the intermediate screens of the application. It is currently unknown whether this behavior could be corrected by learning how to properly use the terminal or whether it is due to psychomotor deficiencies.

The evaluation also highlighted that some older people anticipated the deployment of the system in the " $\mathrm{K}$ " home. For instance, some remarks were related to the concrete implementation of the system and potential coordination difficulties (e.g. "I am afraid that the crowding of residents in the lobby, where the machine will be installed, can be challenging and time consuming"). In fact, it is easy to tackle such an issue by installing several terminals (which is indeed planned but it was not communicated to the subjects). Nevertheless, this type of feedback is valuable. On one hand, such comments help to determine the appropriate settings to install the new system. On the other hand, they show that some older people understand the direct but also the indirect consequences of the deployment of the new system. Taking these elements into account at the deployment stage will probably increase the degree of acceptance of the new technology.

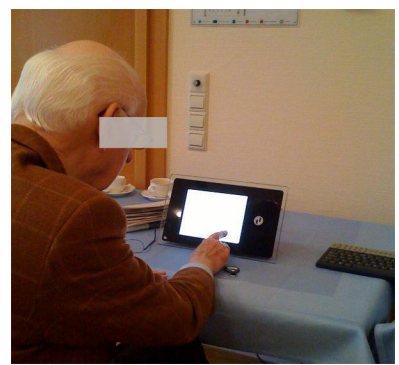

Figure 3: Formative evaluation with a home resident

Another notable finding was the fact that some subjects expressed fears that were not related to their own person. For instance, some participants expressed some skepticism concerning the suitability of this device for some specific residents in the retirement home - especially for persons showing cognitive, sensory and motor impairments. This element indicates that some social factors play a role in the acceptance of the new system. Some older people seem to consider the acceptance of the system at the group level rather than at the individual one. In the longitudinal study planned at a later stage of the project the following hypothesis will be worth investigating: "Are some residents who do not face any problem to use the new technology reluctant to use it because they know that others don't have the sufficient skills to adopt it?"

It is also interesting to point out that some comments did not really refer to the prototype itself but to the management of the home. For instance, some subjects proposed that the lunch menu selection should not be limited to the selection of either one menu or another, but rather subdivided in starters, main dishes and desserts, which would allow a more individual menu composition. This option do not represent a real challenge from a technological viewpoint but it illustrates that the old paper forms used to reserve a menu do not really take into account all the needs of the residents nor perfectly fits the field reality (some residents explained that they already compose their own menu with the waiter of the cafeteria).

Finally some residents suggested to extend the scope of the new system, e.g. to announce in-house activities (e.g. playing cards, scrabble) or to organize meetings with other residents. This element was very important for us because it confirmed the results of the initial requirement analysis phase with the " $\mathrm{K}$ " staff. The system is basically designed to handle the registration process to any type of activity. The database underlying the system is already ready to store the related data. However, at this stage of the project, only the module for reserving the lunch menus is available in the user interface. This feedback from the resident shows that considering a generic problem larger than the menus reservation was a good strategy (cf. KDR10). This finding reinforces our impression that the system can play a social role in the home community. This aspect will be assessed in the longitudinal study.

\section{CONCLUSIONS AND PERSPECTIVES}

This paper reports the first findings of a project aiming to explore the acceptance of new ICT technologies by the older people. More specifically, we discuss the design process of a 
new ICT system to manage the social activities of the residents of a retirement home. Ten key factors are listed and we show how their influenced the design process of the new system. A formative evaluation with a subset of real users has pointed out the overall acceptance of the new system. This evaluation also highlighted the importance of the social factors in the perception of the new technology by older people. These preliminary findings will be completed in the next stage of the TIVIPOL project when the system will be fully deployed in the pilot retirement home. The adoption and the effective usage of the new system will then be monitored. A longitudinal study will be carried out through field observations, qualitative interviews, and system logs analysis. In this context we aim to analyze further the emotional and social factors underlying the acceptance level of this new system. For instance, we might observe the emergence of key residents promoting or discrediting the system. We could also study whether the terminals become social attractors where people meet and discuss about the activities (or other subjects not related to them).

\section{ACKNOWLEDGMENTS}

The research work described in this paper was supported by a grant of the National Research Fund of Luxembourg in the context of the TIVIPOL project. We also thank the manager, the staff and the residents of the ' $\mathrm{K}$ ' home where this study has been carried out.

\section{REFERENCES}

[1] Kientz, J., Patel, S., Jones, B., Price, E., Mynatt E. and Abowd G. The Georgia Tech Aware Home. In Proceedings of the SIGCHI Conference on Human Factors in Computing Systems (Florence, Italy, April 5-10, 2008). CHI '00. ACM Press, New York, NY, 3675-3680.

[2] Mynatt, E., Rowan, J., Craighill, S. and Jacobs, A. Digital family portraits: Providing peace of mind for extended family members. In Proceedings of the SIGCHI Conference on Human Factors in Computing Systems (Seattle, WA, USA, March 31-April 4, 2001). CHI'01, 333-340.

[3] Hagen, I., Cahill, S., Begley, E., Macijauskiene, J., Gilliard, J., Jones, K., Topo, P., Sarikalle, K., Holthe, T. and Duff, P. Assessment of usefulness of assistive technologies for people with dementia. Association for the Advancement of Assistive Technology in Europe (AAATE), Lille, France, 2005.

[4] Kriglstein,S., Wallner,G. HOMIE: An Artificial Companion for Elderly People, In Proceedings of the SIGCHI Conference on Human Factors in Computing Systems (Portland, OR, USA, April 2-7, 2005). CHI'05, 2094-2098.
[5] Mamykina, L., Mynatt, E. and Kaufman, D. Investigating Health Management Practices of Individuals with Diabetes. In Proceedings of the SIGCHI Conference on Human Factors in Computing Systems (Montreal, Québec, Canada, April 22-27, 2006). CHI'06, 927-936.

[6] Perakis,K., Haritou, M., Stojanovic, R., Asanin, B. Koutsouris, D. Wireless patient monitoring for the eInclusion of chronic patients and elderly people. In Proceedings of the $1^{\text {st }}$ International Conference on PErvasive Technologies Related to Assistive Environments, (Athens, Greece, July 15-19, 2008). PETRA'08.

[7] Bhachu, A. S., Hine, N. and Arnott J. Technology Devices for Older Adults to Aid Self Management of Chronic Health Conditions. In Proceedings of $10^{\text {th }} A C M$ Conference on Computers and Accessibility (Halifax, Nova Scotia, Canada, October 13-15, 2008). ASSETS'08, 59-66.

[8] Bouma, H., Fozard, J.L., Bouwhuis, D. G. and Taipale, V. Gerontechnology in Perspective. Gerontechnology, 6(4), 2007, 190-216.

[9] Charness, N. and Schaie, K. W. (Eds.), Impact of Technology on Successful Aging. New York: Springer, 2003, 15-28.

[10] Sustar, H. and Zaphiris, P. Emotional interaction as a way of communication. In Proceedings of the International Conference on Designing Pleasurable Products and Interfaces, (Helsinki, Finland, August 22-25, 2007). DPPI'07.

[11] Davis, F. D. Perceived usefulness, perceived ease of use, and user acceptance of information technology. MIS Quarterly, 13(3), 1989, 319-340.

[12] Venkatesh, V., Morris, M.G., Davis, G.B., and Davis, F.D. User acceptance of information technology: Toward a unified view. MIS Quarterly, 27(3), 2003, 425-478.

[13] Sachpazidis I. And Sakas G. Medication intake assessment. In Proceedings of the $1^{\text {st }}$ International Conference on PErvasive Technologies Related to Assistive Environments, (Athens, Greece, July 15-19, 2008). PETRA'08.

[14] Dickinson, A., Eisma, R. and Gregor, P. Challenging Interfaces / Redesigning Users. In Proceedings of ACM Conference on Universal Usability (Vancouver, British Columbia, Canada, November 10-11, 2003), CUU’03, 6168.

[15] Genov, A., Keavney, M. and Zazelenchuk, T. Usability Testing with Real Data. Journal of Usability Studies, 4(2), 2009, 85-92. 